\title{
Research on Modern Landscape Design based on Ecological Theory
}

\author{
Xiaoqiang Zuo \\ Chongqing College of Electronic Engineering, Chongqing, 401331
}

Keywords: Ecological Theory, Modern Landscape Design, Cultural Prospect

\begin{abstract}
Landscape design began to form in the last century, in the gradual development process has become increasingly mature. In the context of the current serious environmental problems, it is particularly important to strengthen the ecology of landscape design. The combination of ecology and modern landscape can help promote the development of modern landscape design. By strengthening the ecological research of modern landscape design from the theoretical level, we can provide theoretical support for the development of modern landscape design.
\end{abstract}

\section{Introduction}

Ecology is a discipline that is familiar to people, based on the scientific model of classical physics. Ecology is a discipline about the relationship between man and nature, and in a more complex way of observing the life structure of the earth and it is a way to find an organism on all earth as a whole with an inner connection the opinion of. From the beginning of the 19th century, ecology was isolated from the field of pure biology, and the phenomenon of research often touches the essence of people's perception, such as aesthetics, morality, ethics, and economics. So, ecology is a multi-disciplinary discipline. Ecology because of its comprehensive and theoretical guidance significance and become a ubiquitous science of today's society, also affect our landscape design.

\section{Ecological Theory}

Traditional agricultural civilization and industrial civilization are often at the expense of the natural environment in exchange for economic and social development, which makes the human face of ecological crisis. Resource shortage, lack of energy, land desertification, environmental pollution and ecological imbalances become increasingly serious, a large number of biological species tend to extinction. The survival and development of mankind itself is a huge challenge. All this makes people realize that there is only one earth, the earth has a certain limit on the ecological load, to get rid of the current ecological crisis, not only to control and control the current existence of various pollution, but also to fundamentally change the use of resources, as far as possible the effective conversion of resources into products and can be recycled to completely reduce and get rid of waste pollution, so that economic, ecological and social benefits of internal unity. The social ideal of ecology is a new form of human civilization formed under such conditions. Its basic characteristic is the complete solution to the ecological and environmental problems in the era of agricultural civilization and industrial civilization. It is between man and nature. Harmony, it has been able to become a new civilization, because people demand the way and type and type of society to meet the needs of members of the way have undergone a fundamental ecological change. In this society, mankind still has the modern economic industry classification of agriculture and industry, but they will use the principle of ecological organization of production.

Ecologists put forward the idea of ecological control and moderate development, that human beings should control the kind of excessive intervention in the natural environment behavior. Human activities should have a certain limit. It can not exceed the anti-interference ability of an ecosystem, beyond this limit is immoral. Deep ecology puts forward that ecosystems are not only human beings, but other beings have intrinsic values and have the right to survive. Broadly 
speaking, landscape and various environments have the right to keep themselves. Now the ecological ethics of a series of slogans "means frugal, rich purpose", "alive also let others alive", "let the river enjoy flowing", "gently walk on the earth" and other ideas deeply affect the landscape design.

\section{Ecological Ecology and Modern Landscape Design}

In the 18th century, the industrial revolution and early urbanization resulted in a population-intensive, isolated environment that was completely isolated from nature, which attracted the attention of some sociologists. In order to introduce nature into the city, at the same time by the Chinese natural view, natural landscape garden of the impact of mechanical natural view is slowly to the organic view of the transition, the relationship between man and nature is also slowly transformed into harmonious coexistence, coordinated development of the double Excellent relations, the British natural landscape garden began to form and soon prevailed. But it only changed the aesthetic taste of the garden form, did not change the landscape design of the art of the concept. As described by Andrew Jackson, the design of the natural landscape is "the choice of the most beautiful landscape in nature to choose, to remove all the elements of the United States."

Real from the height of the ecological nature of the introduction of the city when the push Oddstede (Frederick Law Olmsted). He is highly respected by the natural landscape. In the form of this garden, he designed at the beginning of his career in Manhattan in 1857, a 2-mile-wide, 0.5-mile-wide city green lung - Central Park. Central Park is located in New York's most prosperous Manhattan downtown, the design of the park first to establish a beautiful natural landscape characteristics of the criteria, focusing on a large area of natural mood, the use of natural layout, the park retained a lot of the original landscape And vegetation, just like a school of mountain forests. Beginning in 1881, he also carried out the design of the Boduten Park system, the park system breakthroughs in the United States urban grid pattern restrictions, rivers, mudflats, grassland to limit the natural space as the basis for delimitation in the city Riverside area to form more than $2000 \mathrm{hm} 2$ ribbon greening, the number of parks into one, forming a series of green space. Known as the "sapphire necklace" of Boduten. These visionary ideas, intended to reconstruct the increasingly loss of urban natural landscape system, effectively promoted the healthy development of urban ecology.

Localized design is the product of the vigorous development of the Midwest after the Civil War. At the end of the 19th century, a group of Midwest Garden Architects, represented by Simonson and Jennifer, created the "Prairie Garden", which embodies a new design concept: the design is not "the form and material of the routine To suit the local landscape, climate, soil, labor conditions and other conditions. " This design is characterized by the use of native plant communities to show local landscape characteristics because of the low cost and the protection of the continuation of the ecological environment, which is widely used in the construction of the nationwide road network by Kolis and Franko, Both sides of the landscaping and slope protection problems.

Local nature exists as a place of land and culture. Especially in China, such a country with thousands of years of farming civilization, native nature attached to the folk color and contains the cultural atmosphere, any city environment can not be replaced. In the real world, native nature and local residents have formed a fit relationship, in addition to the local residents to provide normal living places, but also with a product can be called a small livelihood. That is, the economic considerations are not many, playing the color is very heavy. Local residents in the native nature, the body feel the power of the natural cycle, the four seasons of change and the value of their own existence. At the same time, the native nature of the inherent fast adaptability and has become a modern city of human life and cultural sensibility of a source. City people can use their leisure time, to the local nature to find fun, enrich themselves. Local nature as a secondary natural, its ecological value can not be ignored. For a long time, the formation of native nature and people's production is life closely together, and to be maintained. This ecology belongs to a benign disturbance process. Abandoning the proper management of native nature and ending such disturbances may lead to a crisis in the natural nature of the country itself that has been adapted to the native natural environment, such as many plants or insects, may disappear from this or extinction. 


\section{Principle and Practice of Ecological Landscape Design}

The establishment of the correct relationship between man and nature is respect for nature, the protection of nature, because nature is in its long evolution process, the formation of a self-regulation system to maintain ecological balance. Which water cycle, vegetation, soil, microclimate, terrain in this system play a decisive role. Therefore, in the landscape design, should be adapted to local conditions, the use of the original terrain and vegetation, to avoid large-scale earth renovation project, to minimize the construction of the original environmental impact, as little as possible on the original natural environment changes. Natural ecosystems are endless and tireless, and various conditions and processes are provided to maintain human survival and to meet their needs. Let the natural design work principle emphasize the symbiotic and cooperative relationship between man and nature, through the process of life and the pattern of cooperation, we can significantly reduce the ecological impact of the design.

Try to take simple and efficient measures, the full use of energy and resources and recycling, reduce the consumption of various resources. Multi-choice of local building materials is the classification of waste materials screening, the decay of the magic, both to save raw materials, but also produce another surprising artistic effect.

Modern urban residents are farther and farther away from nature, natural elements and natural processes are increasingly invisible, distant skyline, at the foot of the horizon and the horizon, almost become an abstract term. Children only know the water from the iron pipe out, and from the sink or flush to disappear in the toilet, I do not know where and where to go; busy office workers do not know when the moon is missing, the tide of the tide; People working in the office, even breathing with natural temperature and humidity of the air is a rare thing, not to mention the land of his feet under the soil type, vegetation type and plant species to understand. The nature of the mountains and rivers, birds and beasts, swamp jungle are only TV screen on the screen and the distant nature reserve landscape. As natural processes disappear from the eyes of the public in traditional designs, urban life support systems are often obscured. Wastewater treatment plants, landfills, power plants and substations are intentionally concealed as ugly objects. Natural landscape and process as well as urban life support system structure and process of the blanking, so that people can not care about the status quo and the future of the environment, not to mention the environmental ecology of concern and control the daily behavior of natural landscape and process of blanking People can not care about the status quo and the future of the environment, not to mention the concern for environmental ecology and control the daily behavior. Therefore, to make everyone involved in the design, care of the environment, we must re-reveal the natural process, so that urban residents to re-feel the rain after the skyrocketing, surface runoff sink in the pond; through the leaves of the shaking, feel the natural wind; , See the changes of the four seasons; from the natural leaves of leaves, see the natural decay and degradation process. In the words of Thayer, the landscape is a language that reveals ecology. All this is a way to bring us back to ancient art: visual ecology - a landscape aesthetics that reflects the people's complete dependence on the land system, re-evoking the natural emotional connection between man and nature, in ecology-culture And set up a bridge between the design. The symbiosis between man and the creature is called Wilson "Biophihia", and the attachment relationship between man and land and space is called "Topophiha". It reminds us that humans are designed to adapt and live in nature. The eco-design responds to the attachment of people to the land and the creatures on the land and awakens people's care for nature by revealing and guiding people to experience nature and natural processes. This is a visual ecology (aesthetic visual ecology).

\section{Conclusion}

Landscape design is a comprehensive discipline, ecological problem is not an aspect of landscape design, but throughout the core. Landscape design is to implement the implementation of the need for such as urban design, horticulture and so many theories to guide. Therefore, the "ecology" landscape design discussed in this paper is not to separate the ecology as a separate 
procedure. In fact, every aspect of the landscape ecological design under the guidance of the ecology thought needs to pass through the space and entities to express. We only understand and grasp the basic concept of ecological design and requirements in the design and implementation, there will be more new technologies, new means for our choice, we will do the landscape will be ecological, Practical and beautiful.

\section{References}

[1] Chen Yuezhong, Wang Xiaoxiang. Return to nature, calling for urban green space - red sun ecological garden landscape design [J]. Chinese garden, 2005 (01)

[2] Lu Xiaoyi. Ecological principles in the application of Chinese landscape [J]. Urban and rural construction, 2004 (10)

[3] Yu Kongjian. Land Design: Landscape Science and Art [J]. Planner, 2004 (02)

[4] Deng Yi. The development of urban ecological park and its concept [J]. Chinese Garden, 2003 (12)

[5] Zhang Wenhui, Zhang Lin, Ding Lin. Ecological urban landscape [J]. City, 2003 (06)

[6] Karl Stanness, Huang Guoping. On the principles of ecological planning education [J]. Chinese Garden, 2003 (10) 